\title{
ON THE VANISHING VISCOSITY IN THE CAUCHY PROBLEM FOR THE EQUATIONS OF A NONHOMOGENEOUS INCOMPRESSIBLE FLUID
}

\section{by SHIGEHARU ITOH}

(Received 10 August, 1992)

1. Introduction. Let us consider the Cauchy problem

$$
\left\{\begin{array}{l}
\rho_{t}+\mathbf{v} \cdot \nabla \rho=0 \\
\rho\left[\mathbf{v}_{t}+(\mathbf{v} \cdot \nabla) \mathbf{v}\right]+\nabla p=\mu \Delta \mathbf{v}+\rho \mathbf{f} \\
\operatorname{div} \mathbf{v}=0 \\
\left.\rho\right|_{t=0}=\rho_{0}(\mathbf{x}) \\
\left.\mathbf{v}\right|_{t=0}=\mathbf{v}_{0}(\mathbf{x})
\end{array}\right.
$$

in $Q_{T}=\mathbb{R}^{3} \times[0, T]$, where $\mathbf{f}(\mathbf{x}, t), \rho_{0}(\mathbf{x})$ and $\mathbf{v}_{0}(\mathbf{x})$ are given, while the density $\rho(\mathbf{x}, t)$, the velocity vector $\mathbf{v}(\mathbf{x}, t)=\left(v^{1}(\mathbf{x}, t), v^{2}(\mathbf{x}, t), v^{3}(\mathbf{x}, t)\right)$ and the pressure $p(\mathbf{x}, t)$ are unknowns. The viscosity coefficient $\mu$ is assumed to be nonnegative. In these equations, the pressure $p$ is automatically determined (up to a function of $t$ ) by $\rho$ and $\mathbf{v}$, namely, by solving the equation

$$
\operatorname{div}\left(\rho^{-1} \nabla p\right)=\operatorname{div}\left(\mu \rho^{-1} \Delta \mathbf{v}+\mathbf{f}-(\mathbf{v} \cdot \nabla) \mathbf{v}\right) .
$$

Thus we mention $(\rho, \mathbf{v})$ when we talk about the solution of $(1.1: \mu)$.

The purpose of this paper is to establish the uniform convergence of the solution of (1.1: $\mu)$ with $\mu>0$ to the solution of $(1.1: 0)$ as $\mu \rightarrow 0$. We wish to prove

THEOREM. Assume that

$$
\begin{gathered}
\rho_{0}(\mathbf{x})-\bar{\rho} \in \mathrm{H}^{3}\left(\mathbb{R}^{3}\right) \text { for some positive constant } \bar{\rho} \\
\inf \rho_{0}(\mathbf{x}) \equiv m>0 \text { and } \sup \rho_{0}(\mathbf{x}) \equiv M<\infty \\
\mathbf{v}_{0}(\mathbf{x}) \in H^{3}\left(\mathbb{R}^{3}\right) \text { and } \operatorname{div} \mathbf{v}_{0}=0 \\
\mathbf{f}(\mathbf{x}, t) \in L^{2}\left(0, T: H^{3}\left(\mathbb{R}^{3}\right)\right)
\end{gathered}
$$

and

$$
\mu \leqq 1 .
$$

Then there exists $T^{*} \in(0, T]$ independent of $\mu$ such that the problem $(1.1: \mu)$ has a unique solution $(\rho, v)$ which satisfies

$$
(\rho-\bar{\rho}, \mathbf{v}) \in L^{\infty}\left(0, T^{*}: H^{3}\left(\mathbb{R}^{3}\right)\right) \times L^{\infty}\left(0, T^{*}: H^{3}\left(\mathbb{R}^{3}\right)\right)
$$

and

$$
\mathbf{v}_{\mathbf{x}} \in L^{2}\left(0, T^{*}: H^{3}\left(\mathbb{R}^{3}\right)\right) \text { provided } \mu>0 .
$$

Moreover, let $\left(\rho^{0}, \mathbf{v}^{0}\right)$ be the solution of $(1.1: 0)$ and $\left(\rho^{\mu}, \mathbf{v}^{\mu}\right)$ the solution of $(1.1: \mu)$ with $\mu>0$. Then we have

$$
\sup _{0 \leq t \leq T^{*}}\left[\left\|\left(\rho^{0}-\rho^{\mu}\right)(t)\right\|_{2}^{2}+\left\|\left(\mathbf{v}^{0}-\mathbf{v}^{\mu}\right)(t)\right\|_{2}^{2}\right] \rightarrow 0 \quad \text { as } \quad \mu \rightarrow 0,
$$

where $\|\cdot\|_{k}=\|\cdot\|_{H^{k}\left(\mathbb{R}^{3}\right)}$. 
In the case that $\rho \equiv 1$, we refer to Ebin and Marsden [2] and Ladyzhenskaya [4].

2. Preliminaries. In this section we obtain an a priori estimate for solutions of $(1.1: \mu)$. Let $(\rho, v)$ be a sufficiently regular solution.

Lemma 2.1. If we put

$$
\tilde{\rho}=\rho-\tilde{\rho}
$$

and

$$
\Psi(t)=\int_{0}^{t}\left[1+\|\tilde{\rho}(s)\|_{3}^{2}+\|\mathbf{v}(s)\|_{3}^{2}\right]^{2} d s
$$

then

$$
\sup _{0 \leq s \leq t}\|\tilde{\rho}(s)\|_{3}^{2} \leqq\left\|\tilde{\rho}_{0}\right\|_{3}^{2}+\bar{c} \Psi(t),
$$

where $\tilde{\rho}_{0}=\rho_{0}-\bar{\rho}$ and $\tilde{c}$ is a positive constant depending only on imbedding theorems.

Proof. It follows from $(1.1: \mu)_{1}$ and $(1.1: \mu)_{4}$ that $\tilde{\rho}$ satisfies the equation

$$
\left\{\begin{array}{l}
\tilde{\rho}_{t}+\mathbf{v} \cdot \nabla \tilde{\rho}=0 \\
\left.\tilde{\rho}\right|_{t=0}=\tilde{\rho}_{0}(\mathbf{x}) .
\end{array}\right.
$$

Applying the operator $D^{\alpha}\left(=\left(\partial / \partial x_{1}\right)^{\alpha_{1}}\left(\partial / \partial x_{2}\right)^{\alpha_{2}}\left(\partial / \partial x_{3}\right)^{\alpha_{3}}\right)$ to $(2.4)_{1}$, multiplying the result by $D^{\alpha} \tilde{\rho}$, integrating over $\mathbb{R}^{3}$ and adding in $\alpha$ with $|\alpha|\left(=\alpha_{1}+\alpha_{2}+\alpha_{3}\right) \leqq 3$, then we have

$$
\frac{d}{d t}\|\tilde{\rho}(t)\|_{3}^{2} \leqq \tilde{c}\|\mathbf{v}(t)\|_{3}\|\tilde{\rho}(t)\|_{3}^{2}
$$

Hence, by Young's inequality, it is easy to see that (2.3) holds.

Lemma 2.2. Put

$$
A=1+\left\|\tilde{\rho}_{0}\right\|_{3}^{2}
$$

and

$$
B=\left\|\mathbf{v}_{0}\right\|_{3}^{2}+\int_{0}^{T}\|\mathbf{f}(t)\|_{3}^{2} d t
$$

Then we have

$\|\mathbf{v}(t)\|_{3}^{2}+\int_{0}^{t}\left\|\mathbf{v}_{t}(s)\right\|_{2}^{2} d s+\mu \int_{0}^{t}\left\|\mathbf{v}_{\mathbf{x}}(s)\right\|_{3}^{2} d s \leqq \hat{c}\left[A^{2} B+A(A+B) \Psi(t)+(A+B) \Psi(t)^{2}+\Psi(t)^{3}\right]$,

where $\hat{c}$ is a positive constant depending only on $m, M$ and imbedding theorems.

Proof. We first note that

$$
m \leqq \rho(\mathbf{x}, t) \leqq M,
$$


since we have the representation

$$
\rho(\mathbf{x}, t)=\rho_{0}\left(\left.\mathbf{y}(\tau, \mathbf{x}, t)\right|_{\boldsymbol{r}=0}\right),
$$

where $\mathbf{y}(\tau, \mathbf{x}, t)$ is the solution of the Cauchy problem

$$
\left\{\begin{array}{l}
\frac{d \mathbf{y}}{d \tau}=\mathbf{v}(\mathbf{y}, \tau) \\
\left.\mathbf{y}\right|_{\tau=t}=\mathbf{x} .
\end{array}\right.
$$

(i) We multiply $(1.1: \mu)_{2}$ by $\mathbf{v}$ and integrate over $\mathbb{R}^{3}$. Taking $(1.1: \mu)_{1},(1.1: \mu)_{3}$ and (2.9) into account, we get

$$
\frac{1}{2} \frac{d}{d t}\|\sqrt{\rho} \mathbf{v}\|_{0}^{2}+\mu\|D \mathbf{v}\|_{0}^{2} \leqq M\|\mathbf{f}\|_{0}\|\mathbf{v}\|_{0},
$$

where we use the notation $D^{k} \mathbf{u}=\Sigma_{|\alpha|=k} D^{\alpha} \mathbf{u}$. Multiplying by $\mathbf{v}_{t}$ and integrating over $\mathbb{R}^{3}$ then gives

$$
\begin{aligned}
m\|\mathbf{v}\|_{0}^{2}+\frac{\mu}{2} \frac{d}{d t}\|D \mathbf{v}\|_{0}^{2} & \leqq M\left(\|\mathbf{v}\|_{1}\|D \mathbf{v}\|_{1}\left\|\mathbf{v}_{t}\right\|_{0}+\|\mathbf{f}\|_{0}\left\|\mathbf{v}_{\imath}\right\|_{0}\right) \\
& \leqq c_{1}\left(\|\mathbf{v}\|_{2}^{4}+\|\mathbf{f}\|_{0}^{2}\right)+\frac{m}{2}\|\mathbf{v}\|_{0}^{2}
\end{aligned}
$$

Thus

$$
m\left\|\mathbf{v}_{t}\right\|_{0}^{2}+\mu \frac{d}{d t}\|D \mathbf{v}\|_{0}^{2} \leqq c_{2}\left(\|\mathbf{v}\|_{2}^{4}+\|\mathbf{f}\|_{0}^{2}\right) .
$$

Here and hereafter $c_{j}$ are positive constants depending only on $m, M$ and imbedding theorems.

(ii) Apply the operator $D^{\boldsymbol{\alpha}}$ with $|\boldsymbol{\alpha}|=1$ on each side of $(1.1: \mu)_{2}$, multiply the result by $D^{\alpha} \mathbf{v}$ and integrate over $\mathbb{R}^{3}$. Then, similarly to (i), we get

$$
\begin{aligned}
& \frac{1}{2} \frac{d}{d t}\|\sqrt{\rho} D \mathbf{v}\|_{0}^{2}+\mu\left\|D^{2} \mathbf{v}\right\|_{0}^{2} \leqq c_{3}\left(\|D \rho\|_{2}\|\mathbf{v},\|_{0}\|D \mathbf{v}\|_{0}+\|D \mathbf{v}\|_{1}^{3}\right. \\
& \left.\quad+\|D \rho\|_{2}\|\mathbf{v}\|_{2}\|D \mathbf{v}\|_{0}^{2}+\|D \rho\|_{2}\|\mathbf{f}\|_{0}\|D \mathbf{v}\|_{0}+\|D \mathbf{f}\|_{0}\|D \mathbf{v}\|_{0}\right) \\
& \quad \leqq c_{4}\left(\|D \rho\|_{2}^{4}+\|\mathbf{v}\|_{2}^{2}+\|\mathbf{v}\|_{2}^{4}+\|\mathbf{f}\|_{1}^{2}\right)+\frac{m}{2}\|\mathbf{v}\|_{0}^{2} .
\end{aligned}
$$

If we multiply by $D^{\alpha} \mathbf{v}_{t}$ and integrate over $\mathbb{R}^{3}$, then we have

$$
\begin{aligned}
m\left\|D \mathbf{v}_{t}\right\|_{0}^{2} & +\frac{\mu}{2} \frac{d}{d t}\left\|D^{2} \mathbf{v}\right\|_{0}^{2} \leqq c_{5}\left(\|D \rho\|_{2}\left\|\mathbf{v}_{t}\right\|_{0}\left\|D \mathbf{v}_{t}\right\|_{0}+\|\mathbf{v}\|_{2}\left\|D^{2} \mathbf{v}\right\|_{0}\left\|D \mathbf{v}_{t}\right\|_{0}\right. \\
& \left.+\|D \mathbf{v}\|_{1}^{2}\left\|D \mathbf{v}_{t}\right\|_{0}+\|D \rho\|_{2}\|\mathbf{v}\|_{2}\|D \mathbf{v}\|_{0}\left\|D \mathbf{v}_{t}\right\|_{0}+\|D \rho\|_{2}\|\mathbf{f}\|_{0}\left\|D \mathbf{v}_{t}\right\|_{0}+\|D \mathbf{f}\|_{0}\left\|D \mathbf{v}_{t}\right\|_{0}\right) \\
& \leqq c_{6}\left(\|D \rho\|_{2}^{2}\left\|\mathbf{v}_{t}\right\|_{0}^{2}+\|\mathbf{v}\|_{2}^{4}+\|D \rho\|_{2}^{2}\|\mathbf{v}\|_{2}^{4}+\|D \rho\|_{2}^{2}\|\mathbf{f}\|_{0}^{2}+\|D \mathbf{f}\|_{0}^{2}\right)+\frac{m}{2}\left\|D \mathbf{v}_{t}\right\|_{0}^{2}
\end{aligned}
$$


Therefore

$$
m\left\|D \mathbf{v}_{t}\right\|_{0}^{2}+\mu \frac{d}{d t}\left\|D^{2} \mathbf{v}\right\|_{0}^{2} \leqq c_{7}\left[\|D \rho\|_{2}^{2}\left(\|\mathbf{v}\|_{0}^{2}+\|\mathbf{v}\|_{2}^{4}+\|\mathbf{f}\|_{0}^{2}\right)+\|\mathbf{v}\|_{2}^{4}+\|\mathbf{f}\|_{1}^{2}\right] .
$$

(iii) Adding (2.14) to (2.15), we get

$$
m\left\|\mathbf{v}_{t}\right\|_{0}^{2}+2 \mu \frac{d}{d t}\|D \mathbf{v}\|_{0}^{2}+\frac{d}{d t}\|\sqrt{\rho} D \mathbf{v}\|_{0}^{2}+2 \mu\left\|D^{2} \mathbf{v}\right\|_{0}^{2} \leqq c_{8}\left(\|D \rho\|_{2}^{4}+\|\mathbf{v}\|_{2}^{2}+\|\mathbf{v}\|_{2}^{4}+\|\mathbf{f}\|_{1}^{2}\right) .
$$

Thus, noting that $\mu \leqq 1$, we get

$$
\int_{0}^{t}\|\mathbf{v}\|_{0}^{2} d s+\mu\|D \mathbf{v}\|_{0}^{2}+\|D \mathbf{v}\|_{0}^{2}+\mu \int_{0}^{t}\left\|D^{2} \mathbf{v}\right\|_{0}^{2} d s \leqq c_{9}[B+\Psi(t)] .
$$

(iv) Making use of the operator $D^{\alpha}$ with $|\alpha|=2$ in place of the operator $D^{\alpha}$ with $|\boldsymbol{\alpha}|=1$ and repeating the argument in (ii), we have

$$
\begin{aligned}
\frac{1}{2} \frac{d}{d t}\left\|\sqrt{\rho} D^{2} \mathbf{v}\right\|_{0}^{2} & +\mu\left\|D^{3} \mathbf{v}\right\|_{0}^{2} \leqq c_{10}\left(\|D \rho\|_{2}\left\|D \mathbf{v}_{t}\right\|_{0}\left\|D^{2} \mathbf{v}\right\|_{0}\right. \\
& +\left\|D^{2} \rho\right\|_{1}\|\mathbf{v}\|_{1}\left\|D^{2} \mathbf{v}\right\|_{0}+\|D \rho\|_{2}\|\mathbf{v}\|_{2}^{2}\left\|D^{2} \mathbf{v}\right\|_{0}+\|D \rho\|_{2}\|\mathbf{v}\|_{2}\left\|D^{2} \mathbf{v}\right\|_{0}^{2} \\
& \left.+\|\mathbf{v}\|_{3}\left\|D^{2} \mathbf{v}\right\|_{0}^{2}+\|D \rho\|_{2}\|\mathbf{f}\|_{1}\left\|D^{2} \mathbf{v}\right\|_{0}+\|\mathbf{f}\|_{2}\left\|D^{2} \mathbf{v}\right\|_{0}\right) \\
& \leqq c_{11}\left(\|D \rho\|_{2}^{4}+\|\mathbf{v}\|_{3}^{4}+\|\mathbf{v}\|_{2}^{2}+\|\mathbf{f}\|_{2}^{2}+\|\mathbf{v},\|_{0}^{2}\right)+\frac{m}{2}\left\|D \mathbf{v}_{1}\right\|_{0}^{2}
\end{aligned}
$$

and

$$
m\left\|D^{2} \mathbf{v}_{t}\right\|_{0}^{2}+\mu \frac{d}{d t}\left\|D^{2} \mathbf{v}\right\|_{0}^{2} \leqq c_{12}\left[\|D \rho\|_{2}^{2}\left(\left\|D \mathbf{v}_{t}\right\|_{0}^{2}+\|\mathbf{v}\|_{3}^{4}+\|\mathbf{f}\|_{1}^{2}\right)+\|\mathbf{v}\|_{3}^{4}+\|\mathbf{f}\|_{2}^{2}\right] .
$$

(v) If we add (2.17) to (2.20), then we obtain

$$
\begin{aligned}
m\left\|D \mathbf{v}_{t}\right\|_{0}^{2} & +2 \mu \frac{d}{d t}\left\|D^{2} \mathbf{v}\right\|_{0}^{2}+\frac{d}{d t}\left\|\sqrt{\rho} D^{2} \mathbf{v}\right\|_{0}^{2}+2 \mu\left\|D^{3} \mathbf{v}\right\|_{0}^{2} \\
& \leqq c_{13}\left[\|D \rho\|_{2}^{2}\left(\left\|\mathbf{v}_{t}\right\|_{0}^{2}+\|\mathbf{v}\|_{2}^{4}+\|\mathbf{f}\|_{0}^{2}\right)\right. \\
& \left.+\|D \rho\|_{2}^{4}+\|\mathbf{v}\|_{3}^{4}+\|\mathbf{v}\|_{2}^{2}+\|\mathbf{f}\|_{2}^{2}+\left\|\mathbf{v}_{t}\right\|_{0}^{2}\right] .
\end{aligned}
$$

Hence, due to (2.3) and (2.19),

$$
\begin{gathered}
\int_{0}^{t}\left\|D \mathbf{v}_{t}\right\|_{0}^{2} d s+\mu\left\|D^{2} \mathbf{v}\right\|_{0}^{2}+\left\|D^{2} \mathbf{v}\right\|_{0}^{2}+\mu \int_{0}^{t}\left\|D^{3} \mathbf{v}\right\|_{0}^{2} d s \\
\leqq c_{14}\left[A B+(A+B) \Psi(t)+\Psi(t)^{2}\right] .
\end{gathered}
$$

(vi) Applying the operator $D^{\alpha}$ with $|\boldsymbol{\alpha}|=3$ to $(1.1: \mu)_{2}$, multiplying by $D^{\alpha} \mathbf{v}$ and integrating over $\mathbb{R}^{3}$, then we have

$$
\begin{gathered}
\frac{1}{2} \frac{d}{d t}\left\|\sqrt{\rho} D^{3} \mathbf{v}\right\|_{0}^{2}+\mu\left\|D^{4} \mathbf{v}\right\|_{0}^{2} \\
\leqq c_{16}\left(\|D \rho\|_{2}^{4}+\|\mathbf{v}\|_{3}^{4}+\|\mathbf{v}\|_{3}^{2}+\|\mathbf{f}\|_{3}^{2}+\left\|\mathbf{v}_{t}\right\|_{0}^{2}+\left\|D \mathbf{v}_{t}\right\|_{0}^{2}\right)+\frac{m}{2}\left\|D^{2} \mathbf{v}_{t}\right\|_{0}^{2} .
\end{gathered}
$$


(vii) Add (2.21) to (2.24). Then, due to (2.3), (2.19) and (2.23), we get

$$
\begin{aligned}
& \int_{0}^{t}\left\|D^{2} \mathbf{v}_{t}\right\|_{0}^{2} d s+\mu\left\|D^{3} \mathbf{v}\right\|_{0}^{2}+\left\|D^{3} \mathbf{v}\right\|_{0}^{2}+\mu \int_{0}^{t}\left\|D^{4} \mathbf{v}\right\|_{0}^{2} d s \\
& \leqq c_{17}\left[A^{2} B+A(A+B) \Psi(t)+(A+B) \Psi(t)^{2}+\Psi(t)^{3}\right] .
\end{aligned}
$$

Consequently, it follows from (2.12), (2.19), (2.23) and (2.25) that (2.8) holds.

Lemma 2.3. There exists $T^{*}=T^{*}(\tilde{c}, \hat{c}, A, B) \in(0, T]$ such that

$$
\Psi(t) \leqq 1 \text { for } t \leqq T^{*}
$$

Proof. From Lemma 2.1 and Lemma 2.2, we have a differential inequality

$$
\frac{d}{d t} y(t) \leqq L y(t)^{6},
$$

where $y(t)=1+\Psi(t)$ and $L=\left[\tilde{c} \hat{c} A^{2}(1+B)\right]^{2}$. We conclude that

$$
y(t) \leqq(1-5 L t)^{-1 / 5} \text { provided } t<(5 L)^{-1},
$$

and thus

$$
y(t) \leqq 2 \text { for } t \leqq T^{*}=31 / 160 L .
$$

Because of the above lemmas, the following is easily proved.

Proposition 2.4. There exists a positive constant $c=c(\tilde{c}, \hat{c}, A, B)$ such that

$$
\sup _{0 \leq t \leq T^{*}}\left[\|\tilde{\rho}(t)\|_{3}^{2}+\|\mathbf{v}(t)\|_{3}^{2}\right]+\int_{0}^{T^{*}}\|\mathbf{v}\|_{2}^{2} d t+\mu \int_{0}^{T^{*}}\left\|\mathbf{v}_{\mathbf{x}}\right\|_{3}^{2} d t \leqq c .
$$

3. Proof of Theorem. We first prove the unique solvability of $(1.1: \mu)$. We apply the semi Galerkin method with the basis in $H^{4}\left(\mathbb{R}^{3}\right) \cap J$ provided $\mu=0$ and $H^{5}\left(\mathbb{R}^{3}\right) \cap J$ provided $\boldsymbol{\mu}>\mathbf{0}$, where $J=\left\{\mathbf{u} \in\left\{C_{0}^{\infty}\left(\mathbb{R}^{3}\right)\right\}^{3}: \operatorname{div} \mathbf{u}=0\right\}$. Our approach is completely parallel with that of [1, Chapter 3] without any specific difficulty. To be brief, estimates of the type (2.9) and (2.30) are true for the semi Galerkin approximations and these are sufficient in order to pass to the limit. Hence we can verify the existence of a unique solution of the problem $(1.1: \mu)$ as well as the applicability of the inequalities (2.9) and (2.30) to it. For the detail we refer to [1].

Next we prove $(1.10)$, which is the main result in this paper. If we subtract $(1.1: \mu)$ with $\mu>0$ from $(1.1: 0)$, then we get the following linear system for $\tau=\rho^{0}-\rho^{\mu}$, $\mathbf{w}=\mathbf{v}^{0}-\mathbf{v}^{\mu}$ and $q=p^{0}-p^{\mu}$ :

$$
\left\{\begin{array}{l}
\tau_{t}+\mathbf{v}^{0} \cdot \nabla \tau=-\mathbf{w} \cdot \nabla \rho^{\mu} \\
\rho^{\mu}\left[\mathbf{w}_{t}+\left(\mathbf{v}^{\mu} \cdot \nabla\right) \mathbf{w}\right]+\nabla q=-\rho^{\mu}(\mathbf{w} \cdot \nabla) \mathbf{v}^{0}+\left(\nabla p^{0} / \rho^{0}\right) \tau-\mu \Delta \mathbf{v}^{\mu} \equiv F \\
\operatorname{div} \mathbf{w}=0 \\
\left.\tau\right|_{t=0}=0 \\
\left.\mathbf{w}\right|_{t=0}=0
\end{array}\right.
$$


From this, by proceeding in the same way used for getting a priori estimates, we have

$$
\|\tau(t)\|_{2}^{2} \leqq K_{1} \int_{0}^{t}\|\mathbf{w}(s)\|_{2}^{2} d s
$$

and

$$
\|\mathbf{w}(t)\|_{2}^{2} \leqq K_{2} \int_{0}^{t}\|F(s)\|_{2}^{2} d s
$$

where $K_{1}$ and $K_{2}$ are positive constants depending only on $\sup _{0 \leqq t \leq T^{*}}\left\|\tilde{\rho}^{\mu}(t)\right\|_{3}^{2}$, $\sup _{v \leq t \leq T^{*}}\left\|\mathbf{v}^{\mu}(t)\right\|_{3}^{2}, T^{*}, m, M$ and imbedding theorems.

Let us estimate for the right hand side of (3.3). To begin with, by the usual calculation, we get

$$
\begin{aligned}
& \left\|\left(\rho^{\mu \prime}(\mathbf{w} \cdot \nabla) \mathbf{v}^{0}\right)(t)\right\|_{2}^{2} \leqq\left\|\left(\rho^{\mu}(\mathbf{w} \cdot \nabla) \mathbf{v}^{(0)}\right)(t)\right\|_{0}^{2}+\left\|D\left(\rho^{\mu}(\mathbf{w} \cdot \nabla) \mathbf{v}^{(0)}\right)(t)\right\|_{0}^{2} \\
& \quad+\left\|D^{2}\left(\rho^{\mu}(\mathbf{w} \cdot \nabla) \mathbf{v}^{(0)}\right)(t)\right\|_{0}^{2} \leqq K_{3}\left(M+\|\tilde{\rho}(t)\|_{3}\right)^{2}\left\|\mathbf{v}^{(0}(t)\right\|_{3}^{2}\|\mathbf{w}(t)\|_{2}^{2},
\end{aligned}
$$

where $K_{3}$ is the constant of the theorems of imbedding.

Next, from $(1.1: 0)_{2}$ and (3.2), we obtain

$$
\begin{aligned}
& \left\|\left(\left(\nabla p^{0} / \rho^{0}\right) \tau\right)(t)\right\|_{2}^{2} \leqq K_{3}\left\|\left(\nabla p^{0} / \rho^{0}\right)(t)\right\|_{2}^{2}\|\tau(t)\|_{2}^{2} \\
& \quad \leqq K_{2} K_{3}\left(\|f(t)\|_{2}^{2}+\left\|\mathbf{v}_{t}^{0}(t)\right\|_{2}^{2}+\left\|\mathbf{v}^{0}(t)\right\|_{2}^{2}\left\|D \mathbf{v}^{0}(t)\right\|_{2}^{2}\right) \int_{0}^{t}\|\mathbf{w}(s)\|_{2}^{2} d s,
\end{aligned}
$$

and thus it follows from Proposition 2.4 that

$$
\int_{0}^{t}\|F(s)\|_{2}^{2} d s \leqq K_{4}\left(\mu+\int_{0}^{t}\|\mathbf{w}(s)\|_{2}^{2} d s\right),
$$

where $K_{4}=K_{4}\left(K_{2}, K_{3}, T^{*}, M, c\right)$.

Hence, if we put $K=K_{1} K_{4}$, then

$$
\|\mathbf{w}(t)\|_{2}^{2} \leqq K\left(\mu+\int_{0}^{t}\|\mathbf{w}(s)\|_{2}^{2} d s\right),
$$

and, by Gronwall's inequality,

$$
\|\mathbf{w}(t)\|_{2}^{2} \leqq K \mu\left(K \exp \left(K T^{*}\right)-1\right) .
$$

Now, because of Lemma 2.3 and Proposition 2.4, we find that $K$ and $T^{*}$ are independent of $\mu$, which completes the proof of the theorem.

\section{REFERENCES}

1. S. N. Antontsev, A. V. Kazhikhov and V. N. Monakhov, Boundary value problems in mechanics of nonhomogeneous fluids (North-Holland, 1990).

2. D. Ebin and J. Marsden, Groups of diffeomorphisms and the motion of an incompressible fluid, Ann. Math. 92 (1970), 102-163. 
3. O. A. Ladyzhenskaya, The mathematical theory of viscous incompressible flow (Gordon and Breach, New York, English translation, second edition, 1969).

4. O. A. Ladyzhenskaya, Solvability in the small of nonstationary problems for incompressible ideal and viscous fluids and the case of vanishing viscosity, J. Soviet Math. 1 (1973), 441-451.

Department of Mathematics

FacUlty OF EDUCATION

Hirosaki University

HiRosAKI 036

JAPAN 